G. M. Henkin · J. Leiterer

Andreotti-Grauert Theory by Integral Formulas 
Mathematical Research Mathematische Forschung

Wissenschaftliche Beiträge

herausgegeben von der

Akademie der Wissenschaften der DDR

Karl-Weierstraß-Institut für Mathematik

Band 43

Andreotti-Grauert Theory by Integral Formulas

by

G. M. Henkin

and

J. Leiterer 


\section{Andreotti-Grauert Theory by Integral Formulas}

by

Gennadi Markovič Henkin and

Jürgen Leiterer

Akademie-Verlag Berlin 1988 
Autoren:

Prof. Dr. Gennadi Markovič Henkin

Zentrales Okonomisch-Mathematisches Institut

der Akademie der Wissenschaften der UdSSR, Moskau

Prof. Dr. Jürgen Leiterer

Karl-WeierstraB-Institut für Mathematik

der Akademie der Wissenschaften der DDR, Berlin

Die Titel dieser Reihe werden vom Originalmanuskript der Autoren reproduziert.

ISBN 3-05-5000510-4

ISSN 0138-3019

Erschienen im Akademie-Verlag Berlin, DDR-1086 Berlin.Leipziger Str.3-4 (C) Akademie-Verlag Berlin 1988

Lizenznummer: 202 - 100/433/88

Printed in the German Democratic Republic

Gesamtherstellung: VEB KongreB- und Werbedruck, 9273 Oberlungw1tz

Lektor: Dr. Reinhard Höppner

LSV 1035

Bestellnummer: $7638407(2182 / 43)$

03600 